Interfaces

\title{
Lin et lignes retissés : De la réappropriation de l'histoire dans « Cloth » de Rita Duffy et Paul Muldoon
}

\section{Christelle Serée-Chaussinand}

\section{OpenEdition}

\section{Journals}

Édition électronique

URL : http://journals.openedition.org/interfaces/316

DOI : 10.4000/interfaces.316

ISSN : 2647-6754

Éditeur :

Université de Bourgogne, Université de Paris, College of the Holy Cross

\section{Édition imprimée}

Date de publication : 1 janvier 2017

Pagination : 127-138

ISSN : $1164-6225$

\section{Référence électronique}

Christelle Serée-Chaussinand, «Lin et lignes retissés : De la réappropriation de l'histoire dans «Cloth » de Rita Duffy et Paul Muldoon », Interfaces [En ligne], 38 | 2017, mis en ligne le 13 juin 2018, consulté le 07 janvier 2021. URL : http://journals.openedition.org/interfaces/316 ; DOI : https://doi.org/10.4000/ interfaces.316

\section{(c) (i)}

Les contenus de la revue Interfaces sont mis à disposition selon les termes de la Licence Creative Commons Attribution 4.0 International. 


\title{
LIN ET LIGNES RETISSÉS : \\ DE LA RÉAPPROPRIATION DE L'HISTOIRE DANS «CLOTH » DE RITA DUFFY ET PAUL MULDOON
}

\author{
Christelle Serée-Chaussinand
}

\section{Introduction}

Dans un entretien à la veille de l'inauguration de leur œuvre collaborative " Cuchulain Comforted » au Millennium Art Centre de Portadown en 2007, Paul Muldoon et Rita Duffy réfléchissaient à la valeur du manque et de l'élision dans l'art. ${ }^{1}$ Rita Duffy soulignait, entre autre, son intérêt en tant que peintre pour des vêtements qui, à l'instar des vêtements présentés à l'exposition, n'étaient " plus habités » mais façonnés autour d'un vide ; quelque chose occupait ce vide, expliquait-elle, quelque chose qui pouvait entrer en "résonance » avec l'artiste et l'inspirer dans sa création. ${ }^{2}$ Le poète Paul Muldoon confirmait, quant à lui, l'importance à ses yeux du manque dans les tableaux de Duffy : « en tant que spectateurs, nous pouvons endosser ces vêtements "; " nous pouvons littéralement les réinvestir. Ces tableaux offrent un espace au spectateur, un espace où se glisser, un espace à occuper ${ }^{3}{ }^{3}$ Dans les tableaux de Duffy et le texte poétique de Muldoon qui les accompagne, il n'est ainsi question que d'appropriation ou plutôt d' « investissement » dans la pleine acception du terme : au sens archaïque

1 «Arts Extra », BBC Radio Ulster, 4 Avril 2007.

2 Rita Duffy est née à Belfast en 1959. Son œuvre, à forte dimension autobiographique, aborde une diversité de thématiques tant dans le domaine politique que dans le registre intime. Certaines œuvres, dans une veine féministe et libérale - comme « Mother Ireland » (1988) ou les œuvres de la série « Banquet » (1997) - rendent compte de son expérience de femme dans une société très nettement patriarcale. D’autres comme « Knee-Capping » (1989), «Veil » (2000) ou la série « Outposts » (2006) abordent l'histoire et la politique nord-irlandaise, notamment la violence physique et morale dans la vie quotidienne et en milieu carcéral.

3 Comme Rita Duffy, Paul Muldoon est un homme de Belfast. Né à Portadown, Co. Armagh, en 1951, il a étudié à Queen's University où il s'est associé au groupe des poètes de Belfast aux côtés de Seamus Heaney, Ciaran Carson, Michael Longley et Medbh McGuckian. Il est l'auteur de plus de 30 recueils de poésie parmi lesquels Mules (1977), Meeting the British (1987), Madoc: A Mystery (1990), Horses Latitudes (2006) et plus récemment One Thousand Things Worth Knowing (2015). Sa poésie est brillante et magistralement inventive ; elle se joue des mots et des formes poétiques, conjuguant volontiers culture savante et culture populaire, ciselant les images et multipliant à l'infini les faisceaux de signification. 
de " vêtir, couvrir totalement »; au sens militaire d' " assiéger et s'emparer d'un lieu », au sens plus commun d'« investir ou de s'établir dans un lieu, une situation ».

Dans l'œuvre collaborative de Muldoon et Duffy, il est aussi question de passage de frontière. Les tableaux de Duffy figurant toutes sortes de tissus (du linge de maison, des mouchoirs ou des habits) et la méditation poétique de Muldoon sur son histoire personnelle et familiale en lien avec la " pléiade des vêtements, des chiffons, des toiles et des fleurs de lin » franchissent en effet constamment la ligne de démarcation entre l'intérieur et l'extérieur, le singulier et le collectif, le sacré et le profane, le domestique et le judiciaire, le visuel et le verbal, et s'interrogent ainsi sur les violences du passé et les traumatismes présents; ils tentent également d'exorciser leur peur en mesurant l'impuissance à l'aune du pouvoir, les postures à celle de l'imposture, les élisions aux points de suture. ${ }^{4}$

Je me propose donc d'étudier dans cet article ce qui se tisse entre les tableaux de Duffy et le texte de Muldoon, d'analyser la manière dont chacun exhume le passé, se l'approprie, le révise et redéfinit les contours du paysage politique de l'Irlande du Nord. Il s'agit également de montrer ce qui se joue dans ce dialogue entre texte et image, à savoir la question de la nature du regard.

\section{Icones et histoire}

Parmi les tableaux rassemblés pour l'exposition par Rita Duffy, on trouve une sélection de toiles provenant de la série « Justus » (un ensemble de tableaux représentant les attributs traditionnels d'un juge britannique : col blanc amidonné, perruque en crin de cheval, toge) ainsi que plusieurs œuvres inédites (par exemple, des tableaux figurant des éléments de mobilier, d'autres des mouchoirs blancs). Chaque tableau présente la même caractéristique : le tissu, le vêtement ou l'objet est représenté seul et occupe le centre de l'image, posé au sol ou flottant sur un fond uniforme et neutre à quelques exceptions près où l'arrière-plan est plus élaboré et ressemble à un tissu de camouflage. Ce dispositif pictural attire l'attention sur les détails et participe de l'iconisation de certains objets : ils acquièrent une signification qui excède largement leur sphère habituelle de signification non seulement parce qu'ils sont délibérément isolés mais parce qu'ils sont présentés comme doués d'une aura supplémentaire. Le linge de maison et les meubles du quotidien qui apparaissent dans les premières pages du catalogue

4 Duffy et Muldoon ont vécu directement les troubles en Irlande du Nord et tous deux évoquent ces événements dans leurs œuvres et en l'occurrence dans l'exposition « Cuchulain Comforted » et Cloth, le catalogue qui s'y rapporte. 
ont une valeur sacrée du fait de leur désignation comme « autel », « tombeau » ou « linceul ». A l'inverse, ce dispositif pictural permet de confirmer la valeur mémorielle et hautement symbolique d'autres objets que l'histoire de l'Ulster a transformés. Le mouchoir blanc que Duffy choisit comme motif de plusieurs tableaux n'est pas un simple carré de tissu mais le mouchoir utilisé par le prêtre Edward Daly pour évacuer l'une des victimes de Bloody Sunday sous le feu des balles de l'armée britannique. Le transfert du mouchoir d'un fond neutre à un fond au motif de camouflage confirme, à cet égard, son glissement de la sphère domestique à la sphère politique, du banal à l'iconique (Duffy/ Muldoon, 32-35, 31 et 41). De la même manière, la parka qui figure à la première page du catalogue n'est pas un manteau ordinaire : il appartient à la jeune militante de l'IRA, Mairéad Farrell, qui purgea dix années de prison pour terrorisme, lança un mouvement de protestation ( « dirty protest ») pour obtenir le statut de prisonnier politique et fut exécutée par des agents des services britanniques à Gibraltar en $1988 .{ }^{5}$ Représenter ce vêtement revient ainsi à représenter une relique et permet à Duffy de souligner l'engagement des femmes dans le conflit nord-irlandais. Enfin, la toge et la perruque d'avocat représentés dans le tableau « Objection » (Duffy/Muldoon 29) sont imprégnés de l'histoire de celui à qui ils appartiennent : le conseiller (Queen's Counsel) Philip Magee est connu en effet pour avoir refusé, en 1995, de prêter allégeance à la reine. En choisissant ce vêtement particulier, Duffy pointe ainsi les lignes de fracture et les conflits d'intérêts qui affectent le système judiciaire de territoires tels que l'Irlande du Nord et qui préemptent - voire paralysent - toute décision de justice. Incidemment, on notera que la parka de Mairéad Farrell ou la toge de Magee ne sont pas suspendus sur un cintre ou posés à plat sur la toile comme le reste des vêtements présentés dans l'exposition : ils semblent au contraire façonnés autour de corps fantomatiques, comme si leur propriétaire venait à l'instant de les quitter et pouvait s'y glisser à nouveau pour reprendre cette posture et un geste qui a valeur de geste fondateur.

Le poème de Paul Muldoon, intitulé « Glad Rags », a été quant à lui composé spécialement pour cette collaboration. Les images ont précédé le texte puisque Muldoon a pu découvrir les œuvres de Duffy avant de se lancer dans la rédaction. Constitué de dix paragraphes en prose et multipliant les variations de mise en page, de taille de caractères ou de couleur (Duffy/Muldoon 36), ce poème est marqué par sa très grande iconicité. Nettement autobiographique, le récit de Muldoon se fonde sur son histoire personnelle et familiale. Il forme d'ailleurs une boucle quasi parfaite sur lui-même,

5 Duffy a découvert cette parka au Musée de l'Histoire du Nationalisme Irlandais à Conway Mill (Belfast) où sont rassemblées toutes sortes d'objets liés à la lutte pour l'indépendance irlandaise. On y trouve notamment la reconstitution à l'identique d'une cellule de la prison pour femmes de Armagh, là-même où Mairéad Farrell a été détenue. Le titre du tableau - «Relic » - s'est imposé à Duffy lorsqu'elle a constaté la vénération quasi religieuse qu'inspirait ce vêtement. 
puisqu'il débute sur l'espace blanc de la maternité où est né le poète et se referme sur l'espace blanc (lui aussi) de la salle d'exposition où son œuvre est présentée aux côtés de celle de Duffy - lien métonymique entre le passé et le présent ; franchissement métaphorique de la frontière entre la vie et l'art. A l'instar de Duffy, Muldoon interroge l'histoire de l'Irlande du Nord dans toute sa complexité, sélectionnant une poignée d'événements marquants qu'il isole chacun sur une page unique pour mieux s'en saisir et les faire ressortir textuellement. Il évoque ainsi la prospérité économique de Belfast liée à la culture et à la manufacture du lin dans les années 1920 et 1930 ; plusieurs épisodes sanglants (Bloody Sunday, l'assassinat de Denis Mullan par le gang de Glennane, le massacre de Kingsmill) mais aussi la signature de l'Accord du Vendredi Saint et la vie politique dans l'après-conflit. Chaque événement est systématiquement abordé sous un angle très personnel suggérant que le collectif a des répercussions sur l'individuel et l'affecte intimement : Muldoon ravive le souvenir visuel qu'il a gardé des « barons du lin » de Portadown, les Cowdys, dont la suffisance et la réussite sociale transparaissaient dans la taille impressionnante de leurs chevaux (« impossibly high horses »); il pense au choix de son père de cultiver la fleur de lin ; il insiste sur la proximité de chacun des attentats qu'il ressuscite en décrivant la sorte de chaîne humaine le long de laquelle la violence barbare s'est réverbérée jusqu'à lui : Denis Mullan était le neveu de Maura MacParland, une voisine et amie d'enfance ; il prenait un verre au pub avec Michael Longley le soir du massacre de Kingsmill et a échangé avec l'équipe de journalistes qui venait de couvrir l'événement; il est ami avec une pointure (« a big wig ») de la justice issue des accords de paix, un Cowdys de la famille des Cowdys de son enfance. Dans son poème, Muldoon insiste aussi sur le sectarisme et les divisions communautaires au sein de la société irlandaise mais de manière détournée en s'intéressant aux figures ambivalentes ou transgressives. Ici, il fait astucieusement usage du terme « swaddler » qui désigne en première instance l'emmaillotage d'un enfant, c'est-à-dire son enveloppement dans un lange, pratique qui vise à le protéger mais contraint aussi grandement ses mouvements. Mais dans le contexte nord-irlandais, ce terme est aussi une insulte que les catholiques utilisent pour désigner un protestant. Là, il fait référence à sa mère dont les choix n'ont jamais été motivés par le respect de la tradition ou des impératifs communautaires : elle a délibérément opté pour un accouchement dans une maternité moderne plutôt qu'à la maison ; elle choisissait ses commerçants sans se préoccuper de leurs convictions religieuses :

My mother had no time for what would have been the natural thing to do- for a Catholic to have time only for Catholic doctors, chemists and car dealers. [...] our doctor was a Quaker, our chemist a Quaker, even our car dealer a Quaker. [...] My mother also disdained natural materials, preferring rayon to linen, formica to pine. (Duffy/Muldoon 20, 24, 26) 


\section{Intermédialité}

Un dialogue s'installe ainsi clairement entre les images de Duffy et les mots de Muldoon, placés invariablement sur la page de gauche. La liste des concordances est longue : la référence au juge Robert Cowdy chez Muldoon voisine avec la représentation d'une perruque de magistrat chez Duffy ; un mouchoir blanc est représenté en vis-à-vis d'une évocation de Bloody Sunday ; la réflexion poétique de Muldoon sur l'étymologie de «parka » (désignant une peau d'animal en langage esquimau), sur les « éraflures » (« excoriation»), les pelures (« unpeeling ») et sur l'origine commune de « flax » (le lin) et de «flay » (« écorcher ») fait doublement écho au mot « flak » qui sert de titre à la représentation d'un gilet pare-balles par Duffy. On trouve même un exemple de contamination entre le poétique et le pictural lorsque la phrase nominale «Arterial blood » est imprimée dans le même ton rouge-sang que la soutane de cardinal à droite dans l'œuvre de Duffy : l'effet de coulure accentué vers la base du tableau donne à penser que celui-ci a déteint sur le texte. ${ }^{6}$ Cependant, ni le texte ni les tableaux ne sont la stricte illustration du contenu dans l'autre médium. Ils existent autant séparément qu'à travers leur juxtaposition. On retrouve en l'occurrence ici un jeu texte/image analogue à celui qui se jouait déjà dans Plan B, un recueil que Muldoon a composé en collaboration avec le photographe écossais Norman McBeath. Dans la préface, le poète remarque que ses poèmes et les images de McBeath établissent « toutes sortes de connexions qui leur sont propres, aucune n'étant évidente [...] elles sont néanmoins d'une certaine manière toutes significatives, toutes accompagnées de petits grognements, les sourires et grimaces de la reconnaissance ». $^{7}$ Il en va de même dans Cloth.

Il n'en reste pas moins que le texte de Muldoon est d'une grande picturalité : certaines idées abstraites sont imagées (la supériorité des Cowdy figurée par la taille de leurs chevaux) ; la couleur est partout présente ; toutes sortes de textiles (la toile, la rayonne, la batiste, la mousseline, le lin, etc.) sont mentionnés par le poète qui pratique, selon ses propres termes, une " autopsie de la fibre » (« the forensics of the thread », Duffy/Muldoon 30), trahissant sa prédilection pour les textures et les mots qui les décrivent et proposant une façon d'ekphrasis de la pratique picturale de Duffy qui repose sur un travail minutieux au cœur de la fibre et de la trame des étoffes, et en particulier des étoffes de lin.

6 On en peut s'empêcher de voir dans cet effet de coulure un lointain rappel du travail de la couleur dans les portraits du pape Innocent $\mathrm{X}$ réalisés par Francis Bacon.

7 Paul Muldoon, Plan B, London, Enitharmon Press, 2009. 
Cette œuvre collaborative qui associe visuel et verbal autour de la question du conflit nordirlandais conduit ainsi à une révision et à une ré-interprétation du passé en même temps qu'elle se ré-approprie le présent. Muldoon et Duffy s'emparent d'éléments dans leur environnement immédiat passé ou présent - et invitent le lecteur-spectateur à re-considérer les violences d'hier, les traumatismes et les divisions d'aujourd'hui, et à envisager les voies possibles de la (ré)conciliation. La représentation par Duffy d'attributs symboliques du pouvoir - une veste de police, une tenue d'avocat ou de magistrat, un habit de cardinal ou le gilet pare-balles d'un paramilitaire-dénonce les nombreuses postures et impostures des hommes de pouvoir et pointe leur terrible responsabilité. Inversement, ses images de nappes si propres et si bien ajustées suggèrent une dérisoire maîtrise cantonnée à la sphère domestique et une impuissance réelle dans la sphère politique. A travers ces deux séries de tableaux, et en résonance avec une culture où les lieux ont un genre, ce sont aussi le masculin et le féminin qui sont mis en regard, à l'instar de ce que l'on observe dans la seule photographie de l'exposition, intitulée « Dessert », qui associe de manière résolument surréaliste un moulage de mitraillette en chocolat et un délicat napperon en damassé de lin rehaussé de dentelle fine (Duffy/Muldoon 47). De la même manière, lorsqu'il associe souvenirs personnels et souvenirs collectifs ; morts sanglantes et naissances dans des espaces immaculés ; " clichés » verbaux et « clichés » visuels ; lorsqu'il attire notre attention sur de singulières proximités étymologiques, Muldoon nous invite à ré-évaluer les destructions liées au conflit et les efforts de reconstruction, tant au niveau institutionnel que judiciaire, suggérant qu'il est difficile de s'abstraire du passé mais qu'il est nécessaire de vivre dans le présent. La vision artistique de Muldoon et Duffy intègre donc une révision politique et historique. Toutefois, au-delà de ces considérations nonesthétiques, leur travail conduit à une réflexion sur la nature du regard.

\section{Elision, suture et regard}

En effet, l'un des aspects particuliers du travail de Duffy dans « Cuchulain Comforted » est de peindre des textiles familiers d'une manière qui n'est pas familière. Elle isole et met en scène chaque mouchoir, chaque perruque, chaque col ; elle théâtralise la représentation de chaque vêtement, de chaque linge, soit qu'il flotte au milieu d'une sorte de néant, soit qu'il se trouve posé à même le sol. Ce faisant, elle attire délibérément l'attention du spectateur sur la matérialité et la singularité des différentes étoffes : le regard du spectateur est capté par les entrelacs et les plis, par les nombreuses nuances de gris, de blanc, de noir et de rouge, par la multitude des textures (étoffe douce au toucher ou rêche, délicate ou pare-balles, transparente ou opaque, claire ou de couleur foncée). Duffy joue enfin sur certains titres de manière à renforcer les effets de trompe-l'œil ou les détournements qu'elle 
concocte par l'entremise de son pinceau : les cols amidonnés sont appelés tour à tour "écharpe » or « fer à cheval » mais sont peints comme s'il s'agissait de coiffes féminines, et plus précisément des coiffes traditionnelles Quaker. La signification profonde d'une telle pratique picturale est, entre autre, de marquer la contingence et la relativité de tout regard et de toute interprétation : les objets changent en effet d'apparence suivant l'angle depuis lequel on les observe ou suivant la face qu'ils nous présentent. De même, la diversité des contextes, des tonalités, des textures renvoie métaphoriquement à l'importance des détails; notre perception est susceptible d'être modifiée par le contexte, la distance par rapport à l'objet, l'intensité de la lumière ou la part d'ombre sur lui. On peut, bien entendu, en première lecture, percevoir la dimension politico-historique de ce travail sur la représentation et les conditions du voir : comme le regard, toute recherche de la vérité sur les exactions et les violences passées dans le contexte nord-irlandais est soumise à de nombreuses contingences : éloignement dans le temps des événements, témoignages discordants selon le degré d'implication des témoins et leur positionnement par rapport à cet événement, archives et preuves donnant lieu à de multiples interprétations, etc. De même, le processus de paix dépend du regard que les deux communautés portent sur le passé et de la manière dont elles construisent, sur cette base, le présent. Mais au-delà de ce premier degré de lecture, on notera que, de toile en toile, s'élabore une réflexion non seulement esthétique mais aussi philosophique sur le regard. La pratique artistique de Duffy semble en effet renvoyer à cette dimension particulière de l'art que Lacan désigne dans son essai consacré à la schize de l'œil et du regard, à savoir que tout tableau est un " piège à regard " (Lacan 102). A l'instar du tableau de Holbein que Lacan prend pour exemple («Les Ambassadeurs »), tous les objets peints par Duffy nous regardent cependant que nous les voyons : il y a une part de leurre et de dépossession dans les images (Lacan 116-9) qui nous renvoient en tant que spectateur à notre condition de sujet et ce faisant, à notre condition de sujet « regardé » (Lacan 87) et de sujet fondamentalement divisé ou faillé (Lacan 96-7).

A cet égard, il est intéressant de remarquer que Duffy insiste aussi beaucoup sur les élisions et les éclipses de même que sur les sutures, les coutures et les objets qui servent à accrocher ou à nouer. L'absence des corps dans ses tableaux peut être vue comme la présence « en creux » de toutes les victimes du conflit, de tous ceux qui ont péri et ont disparu prématurément. " Elision » qui compte parmi les tableaux les plus expressifs de l'exposition et du catalogue est particulièrement frappant à cet égard (Duffy/Muldoon 11). Il représente une chemise blanche, figée autour d'un corps manquant et flottant sur un fond à double trame : esquisses à l'encre noire d'armes de poing et de fusils se détachant sur une imitation de tissu de camouflage. Inversement, Duffy montre une prédilection pour les systèmes d'accroche et nouage comme si elle voulait signifier l'importance et l'absolue nécessité de recréer du lien, de réparer et de reconstruire : cintres pour suspendre un vêtement prenant ici des airs de squelettes 
symboliques à la tête recourbée et au cou étranglé de fer ; coutures, boutons et boutonnières ; rubans et cordelières pour fixer un col ; étoffes ou vêtements gansés de dentelle. Tous ces éléments participent aussi du rôle que Duffy assigne à ses œuvres : tenter d'articuler visuellement l'inarticulable...

La rétrospective poétique de Muldoon se fonde sur une même pratique de défamiliarisation que chez Duffy, recèle un même désir de couture et de suture et donne une même leçon sur le regard. Dans "Glad Rags », comme dans nombre d'autres œuvres du poète, les néologismes sont légion. Les combinaisons bizarres qu'il effectue ici- « chocolutherans » or « chocatholics » (Duffy/Muldoon 24) ou encore " chokalashnikov » (Duffy/Muldoon 2007, p. 44) — ne sont rien d'autre que des mots coupés et cousus ensemble. Dans son propre univers - celui du langage et des mots - le poète suggère ainsi que les distinctions et les ségrégations sont affaire de définition et d'appellation. Les divisions sectaires peuvent être littéralement créées ou éradiquées, littéralement décrétées ou niées. A un détail près toutefois : les mots-valises que Muldoon inventent ne sont pas seulement des néologismes, ce sont aussi des barbarismes et en cela ils rappellent la part inhérente de non-sens et de violence dans le langage et dans l'histoire. Muldoon insiste aussi sur l'importance des vêtements que nous revêtons. Ce sont plus que de simples attributs : ils nous protègent, ils nous cachent, ils modifient notre apparence et parfois notre statut. En d'autres termes, ils nous investissent tout autant qu'ils nous revêtissent. Ils sont chargés de symbole ; ils incarnent le pouvoir ou trahissent une impuissance. Le clergé, dont on dit que les membres « prennent ou portent l'habit», est un exemple flagrant de cette valeur particulière du vêtement : «l'habit fait ou ne fait pas le moine » dit l'adage. Il en va de même pour les magistrats que l'on désigne parfois par l'expression « hommes de robe». Muldoon induit que dans d'autres contextes, tels que le contexte nord-irlandais à l'époque du conflit ou du processus de paix, les vêtements jouent aussi un rôle essentiel. Suivant que l'on porte la tenue paramilitaire, l'habit de juge ou la couverture souillée d'un prisonnier politique, une croix catholique ou un insigne orangiste, la donne est bien différente. Dès lors, pour avancer et dépasser les divisions passées, il convient de se défaire de tous ces oripeaux superficiels (drapeaux, emblèmes, etc.) ; il faut se débarrasser du linge sale (« dirty linen »), changer d'habits et se revêtir de ses plus beaux atours ("glad rags »). L'esprit de clan exacerbé doit céder le pas à la politique inter-communautaire. C'est en ce sens que le poète écrit :

No washing of dirty linen in public. No flags or emblems. [...] All about excoriation. All about the unpeeling. [...] All about the unpeeling. The shrugging off of the outer layers. The retting. The scutching. The bleaching on the bleach greens of Stormont. Our glad rags. Put our best foot forward. (Duffy/Muldoon 28 and 42-44) 
Tout en affirmant cela, Muldoon admet toutefois indirectement que ce processus de dépouillement est extrêmement difficile. Il s'attarde sur l'étymologie du mot «Parka » dont il rappelle qu'il s'agit d'un mot esquimau (aléoutien) signifiant « peau » pour souligner qu' on change de vêtements mais qu'on ne change pas de peau. Il signale aussi la violence inhérente à cette sorte de deuxième peau que sont les vêtements : «flax » est étymologiquement associé au verbe « to flay » qui signifie « dépecer ou écorcher ». Le lecteur est ainsi amené à réfléchir à ce qu’il en coûte de se défaire du passé.

La réflexion engagée sur la nature et la valeur du regard est enfin menée à travers la série de titres qui désignent le travail de Duffy et Muldoon et en dessinent les contours, à la fois multiples et protéens. Le titre du projet global mené par le Millennium Court Arts Centre— « Interrogating Contested Spaces in Post-Conflict Society »-met l'accent sur la dimension critique de cette ouvre collaborative. A travers leur dialogue, les images de Duffy et le texte de Muldoon deviennent « un espace où les postulats du processus de paix sont soumis à une réflexion critique, où la notion même de paix et ses conditions de possibilité sont examinées. [...] [Ces tableaux et ce texte] explorent la temporalité complexe d'un présent qui s'écrit dans le but avoué de s'abstraire du passé $»{ }^{8}$ (Bell 28-9) Ils abordent ainsi toutes sortes de questions : la justice dans la période de transition, l'abandon des armes, la division communautaire, la peur, l'espoir, etc. Le titre propre à l'exposition- « Cuchulain Comforted »-fait quant à lui référence au héros celtique qui, selon la légende, aurait caché une blessure mortelle en s'enveloppant dans son vêtement. Mais ce guerrier mythique est une figure hautement ambivalente, célébrée par les Catholiques Républicains comme par les Protestants Unionistes, ces derniers voyant en lui le «chien de l'Ulster » qui défendit valeureusement la province face aux armées de Medb, la reine du Connaught. Ce titre s'entend également en référence à Yeats dont le célèbre poème évoque le destin de Cuchulain outre-tombe : il est accueilli dans le royaume des ombres par une horde de couards qui l'invitent à tisser son propre linceul avec du fil de lin afin d'opérer sa transfiguration ou sa métamorphose, c'est-à-dire afin de s'affranchir de son passé guerrier et violent pour accéder à une forme de paix et de spiritualité :

8 Ma traduction de : « sites in which the assumptions of transition are opened up for critical reflection, probing the notion of what constitutes Peace and its conditions of possibility. [...] [They] explore the complex temporalities of a present self-consciously attempting to narrate itself away from the past ». 
A Shroud that seemed to have authority

Among those bird-like things came, and let fall

A bundle of linen. Shrouds by two and three

Came creeping up because the man was still.

And thereupon that linen-carrier said:

'Your life can grow much sweeter if you will

'Obey our ancient rule and make a shroud;

Mainly because of what we only know

The rattle of those arms makes us afraid.

'We thread the needles' eyes, and all we do

All must together do.' That done, the man

Took up the nearest and began to sew. (Yeats 2000

«Cloth», le titre choisi pour le catalogue accompagnant l'exposition, met enfin l'accent sur la matérialité et la littéralité de cette œuvre collaborative : «tissu » vaut pour tous les textiles représentés par Duffy, pour les toiles qui servent de support à ses tableaux, pour le texte de Muldoon qui se tisse autour de l'histoire du lin et du vêtement. Mais c'est le mot « line » plutôt que " cloth » qui apparaît finalement comme le véritable « fil rouge » de l'œuvre. Mis en exergue par Muldoon page 40, « line » est en effet un terme polysémique qui selon le contexte désigne une ligne, un fil ou un cordon, un trait, un vers de poésie, une lignée, une conduite ou une ligne d'action et de pensée. Si chacune de ces significations éclaire le dialogue intermédial entre les images de Duffy et le texte de Muldoon, ce dialogue est magistralement confirmé et renforcé par l'étymologie : le poète souligne de manière opportune le lien entre « line », « linen » et « flax », matière dont est fait le lin : « Toe the line. Line, from the Latin linum, 'flax' ». Le matériau sémiotique et sémantique dont sont constitués les mots de Muldoon est de même essence que la matière première dont sont faits les vêtements peints par Duffy. 


\section{Conclusion}

En référence à une série de tableaux réalisés en 2006 et représentant des miradors, Rita Duffy remarquait : «Avec le temps, l'immédiateté de la violence, de la suspicion et de la paranoïa s'estompe. Le paysage politique dans le Nord se dessine progressivement selon des contours différents ; il devient un lieu de reconstruction, où la ville et la campagne sont reconfigurées en fonction d'une logique nouvelle fondée sur la nécessité économique ; l'histoire est une fois encore réécrite à l'aune des impératifs du présent. Du fait de ces petits arrangements avec le passé, les histoires d'hier peuvent être racontées de façon différente ; ce qui était enterré est exhumé ; d'anciennes certitudes sont remises en cause. Ces tableaux sont un acte de surveillance et en tant que tels, ils posent la question de la nature du regard ». ${ }^{9}$ Comme cette série de tableaux (« Outposts »), « Cloth » n'édicte aucune vérité absolue, tant sur le plan politique, que moral ou éthique ; aucun jugement définitif n'est prononcé. Liberté est laissée au lecteur-spectateur d'interpréter et de prendre position. Par l'entremise des images et des mots, Rita Duffy et Paul Muldoon exhument le passé, questionnent leurs certitudes, retissent le fil de leur histoire avec la trame de l'Histoire de leur province. Dans cette entreprise, ils nous enseignent que plusieurs perspectives et plusieurs visions sont possibles, que tout est affaire de regard et que tout se joue dans l'écart entre ce que l'on regarde et ce que l'on veut voir.

9 Ma traduction de : «As time passes the immediacy of violence, suspicion and paranoia subsides. Different contours begin to dominate the political landscape of the North; a place of reconstruction, where city and country become reconfigured according to a logic of economic necessity, and history is once more remade in the image of current imperatives. These negotiations with the past permit its stories to be told in a different way; that which was buried becomes exhumed, previous certainties become open to question. These paintings are an act of surveillance, and as such, they are about the whole nature of looking ». Source : " Troubles Archive », http:// www.troublesarchive.com/artists/rita-duffy (accédé le 28 octobre 2016). 


\section{Ouvrages Cités}

BELL, Viki. December 2011. "Contemporary Art and Transitional Justice in Northern Ireland: The Consolation of Form". Journal of Visual Culture. Vol. 10(3). 324-353.

DUFFY, Rita, MULDOON, Paul. 2007. Cloth: A Visual and Verbal Collaboration by Rita Duffy and Paul Muldoon. Portadown, Millennium Court Arts Centre.

LACAN, Jacques. 1973. Le Séminaire - Livre XI : Les quatre concepts fondamentaux de la psychanalyse. Paris, Le Seuil.

MULDOON, Paul. 2009. Plan B, London, Enitharmon Press.

YEATS, William Butler. 2000. Poems Selected by Seamus Heaney. London, Faber.

http://www.troublesarchive.com/artists/rita-duffy (accédé le 28 octobre 2016). 